\title{
pfmdr1 GENOTYPING AND IN VIVO MEFLOQUINE RESISTANCE ON THE THAI-MYANMAR BORDER
}

\author{
AMY L. NELSON, ANNE PURFIELD, PHILIP MCDANIEL, NICHIPAT UTHAIMONGKOL, NILLAWAN BUATHONG, \\ SABAITIP SRIWICHAI, R. SCOTT MILLER, CHANSUDA WONGSRICHANALAI, AND STEVEN R. MESHNICK \\ University of North Carolina School of Public Health, Department of Epidemiology, Chapel Hill, North Carolina; University of \\ North Carolina School of Medicine, Department of Microbiology and Immunology, Chapel Hill, North Carolina; Armed Forces \\ Research Institute for the Medical Sciences, Bangkok, Thailand
}

\begin{abstract}
Molecular markers have been proposed as a method of monitoring malaria drug resistance and could potentially be used to prolong the life span of antimalarial drugs. Single nucleotide polymorphisms (SNPs) in the Plasmodium falciparum gene $p f m d r 1$ and increased gene copy number have been associated with in vitro drug resistance but have not been well studied in vivo. In a prospective cohort study of malaria patients receiving mefloquine treatment on the Thai-Myanmar border, there was no significant association between either pfmdr1 SNPs or in vitro drug sensitivity and mefloquine resistance in vivo. Increased $p f m d r 1$ gene copy number was significantly associated with recrudescence (relative risk 2.30, 95\% CI 1.27-4.15). pfmdr1 gene copy number may be a useful surveillance tool for mefloquine-resistant falciparum malaria in Thailand.
\end{abstract}

\section{INTRODUCTION}

Each year, there are as many as 300 million cases of malaria, causing severe anemia among children, perinatal mortality, and low birth weight among infants, as well as at least 1 million yearly malaria deaths. ${ }^{1}$ Drug resistance has been documented in most malaria-endemic countries, and multidrug resistance is spreading. ${ }^{1}$ Multidrug resistance of the malaria parasite Plasmodium falciparum poses a threat to malaria control in tropical developing countries worldwide.

Traditional methods for detection and surveillance of drugresistant malaria are cumbersome, relying on variable clinical outcomes (in vivo resistance) or on time-consuming laboratory assays (in vitro resistance). ${ }^{2}$ However, new methods for the surveillance of drug-resistant malaria using molecular markers have recently been suggested. ${ }^{3}$ Surveillance mechanisms using molecular markers may be an appropriate technology for middle-income countries such as Thailand.

Polymorphisms in the Plasmodium falciparum gene pfcrt have been strongly correlated with in vitro chloroquine resistance. ${ }^{4-6}$ Studies have reported conflicting results on an association between polymorphisms in the falciparum gene pfmdrl and in vitro resistance to mefloquine. ${ }^{7-16}$ Of these two markers, only pfcrt polymorphisms have been shown to be associated with in vivo resistance (to chloroquine). ${ }^{7}$ Studies are needed to demonstrate whether $p f m d r 1$ polymorphisms are associated with clinical resistance.

In previous work conducted in Southeast Asia, ${ }^{17}$ we found that $p$ fmdr1 polymorphisms (determined on parasite cultures) were strongly related to in vitro mefloquine resistance in cultured malaria isolates from across Southeast Asia. Polymorphisms in pfmdrl clustered into four specific patterns: the "wild-type" (Category I); a Tyr86 polymorphism only (Category II); a Phe184 polymorphism only (Category III); and Phe184 in combination with Cys1034 and/or Asp1042 (Category IV) (Table 1). Isolates in Categories I and III were more sensitive to chloroquine and more resistant to mefloquine, artesunate, and artemisinin than isolates in Categories II and IV. Thus, these categories reflect an expected "mefloquine-resistant" group (Categories I and III combined) and a "mefloquine-sensitive" group (Categories II and IV combined). pfmdrl gene amplification was also strongly associated with in vitro mefloquine resistance.
In the current study, we investigate the relationship between polymorphisms and gene amplification of pfmdr1 and in vivo mefloquine failure in a prospective study of malaria patients on the Thai-Myanmar border.

\section{MATERIALS AND METHODS}

This project received ethical approval from the Thai Ministry of Public Health, the Walter Reed Army Institute of Research (WRAIR), and the University of North Carolina at Chapel Hill.

The current study took place in Sangkhlaburi District, Kanchanaburi Province, Thailand, located near Three Pagodas Pass at the Thai-Myanmar border. The district center is free of malaria transmission. Malaria cases presented to local malaria clinics and hospitals were almost always occupationallyrelated (e.g., migrant workers from Myanmar). Malaria transmission is year-round with a seasonal peak around MaySeptember.

Subject recruitment. We evaluated patients presenting with fever (oral temperature $\geq 38^{\circ} \mathrm{C}$ ) to government malaria clinics and a hospital outpatient clinic in Sangklaburi. Patients were enrolled during July 2001-August 2002. A fingerprick blood smear, part of the standard evaluation of febrile illness, was used to determine malaria status through Giemsa stain and microscopic evaluation. Patients who were age 18 years or more, with $P$. falciparum monoinfection and with parasitemia (asexual forms) $\geq 0.05 \%$ were invited to participate. Patients who were pregnant, who had vivax infection, a history of antimalarial drug use within the past 2 weeks, bleeding tendency (by self-reported history or based on medical records), or severe/complicated malaria, which was defined as any case of malaria emergency treatment of any reason or that where referral for inpatient care was deemed necessary, were excluded. The study was explained in detail by a research nurse or investigator working at the enrollment site, through a translator if Thai was not the primary language of the potential subject.

Data collection. Consenting subjects were interviewed for medical history and asked to donate $8 \mathrm{~mL}$ of blood by venipuncture. A short subject information form that captures demographic data, past malaria experience, malaria treatment, and past illnesses was also completed for each subject. 
TABLE 1

Patterns of gene polymorphisms observed from isolates across Southeast $\mathrm{Asia}^{17}$ (mutated amino acid positions are shown in bold)

\begin{tabular}{lcccc}
\hline & \multicolumn{4}{c}{ Codon position } \\
\cline { 2 - 5 } Category & $\mathbf{8 6}$ & $\mathbf{1 8 4}$ & $\mathbf{1 0 3 4}$ & $\mathbf{1 0 4 2}$ \\
\hline I & Asn & Tyr & Ser & Asn \\
II & Tyr & Tyr & Ser & Asn \\
III & Asn & Phe & Ser & Asn \\
IV & Asn & Phe & Cys & Asp \\
\multicolumn{1}{c}{ or } & Asn & Phe & Cys & Asn \\
\multicolumn{1}{c}{ or } & Asn & Phe & Ser & Asp \\
\hline
\end{tabular}

An aliquot of blood was used to initiate malaria parasite culture immediately at the field laboratory of the Armed Forces Research Institute of Medical Sciences (AFRIMS) in Sangkhlaburi prior to transfer to AFRIMS main laboratory in Bangkok, where the in vitro assays against a panel of antimalarial drugs (mefloquine, chloroquine, and quinine) took place. The rest of the blood samples was stored in liquid nitrogen and transported to AFRIMS in Bangkok for storage at $-80^{\circ} \mathrm{C}$. Patients were treated with a single $750-\mathrm{mg}$ dose of mefloquine and observed for 1 hour.

Follow-up. Volunteers were asked to return for scheduled visits on the following days (given Day $0=$ day of enrollment): Days 3, 7, 21, 28, 35, and 42 or at any time symptoms recurred. A history of ongoing or recurrent symptoms and compliance with the prescribed drug regimen was obtained, and malaria thick/thin blood smear was prepared from a fingerprick. The probability of being reinfected with malaria during the follow-up period was extremely small in the village where this study was conducted. Patients were asked to refrain from entering the jungle, where the risk of infection might be higher. The entomological inoculation rate (EIR) in this area has been estimated to be 1-2 per year. Therefore, if the subject was found positive for $P$. falciparum again within 42 days, this patient was considered "recrudescent" in our analyses.

Eight milliliters of blood was drawn from recrudescent patients and processed as for the initial visit. Recrudescent participants were treated with standard second-line antimalarial regimens following the guidelines of the Thai malaria control program for that region. Recrudescent subjects were followed for 28 additional days with the same scheduled follow-up as outlined above.

Laboratory methods. The in vitro drug sensitivity assay was performed by the radioisotope microdilution technique, slightly modified from that previously described. ${ }^{18}$

To determine genotype of parasite DNA extracted from patient blood, we used a real-time polymerase chain reaction (PCR; ABI Prism 7000 Sequence Detection System, Applied Biosystems, Foster City, CA) assay as previously described. ${ }^{19}$ This assay was used for genotype analysis of four polymorphic sites in pfmdr1: Asn86 $\rightarrow$ Tyr86, ${ }^{10}$ Tyr184 $\rightarrow$ Phe184, Ser1034 $\rightarrow$ Cys1034, and Asn1042 $\rightarrow$ Asp1042. Briefly, fluorescent-labeled minor groove binding (MGB) probes were designed specific to the polymorphic site for wild-type or mutant species. Following amplification, the genotype of four polymorphic sites was determined by increase in fluorescent emission of the wild-type or mutant probes. In one case, DNA extraction from the blood sample did not yield DNA that could be amplified, so DNA from parasite culture was used. pfmdr1 gene amplification was determined using quantitative real-time PCR (ABI Prism 7000 Sequence Detection System) as previously described. ${ }^{17}$ The detection of multiple copies is accomplished by examining the standard curve comparing $p f m d r l$ to $l d h$ in a wild-type, drug-sensitive strain of parasite. Using this method, the ratio of ldh:pfmdrl should always be $1: 1$. The standard curve was run as a control with each experiment to determine gene amplification. Briefly, we designed dual-fluorescent labeled probes specific to $p \mathrm{fmdr} 1$ or $l d h$. We quantified $p f m d r l$ and $l d h$ copy number based on the emission of the probes after real-time PCR amplification.

Analysis. Data were entered into Excel spreadsheets by study staff. Analysis was performed using SAS v8 (SAS, Inc., Cary, NC). Based on previous data, ${ }^{17}$ we grouped $p f m d r 1$ genotypes into "sensitive" strains, containing pfmdrl genotype Categories II and IV (Tyr86; or Phe184 with either Cys1034, Asp1042, or both), and "resistant" strains, containing pfmdr1 genotype Categories I and III (wild type, or Phe184), as shown in Table 1. Some isolates had mixed genotypes. Most often, the potential combinations of genotypes fell into the same broad category; for example, a mixed genotype at position 184 falls into the Category I/III, whether it is wild type (Category I) or polymorphism (Category III). The planned enrollment was 200 patients, with a final sample size of at least 150 needed to detect an association between drug resistance and polymorphisms under the expected distributions. The relationship between molecular markers and in vitro outcomes was assessed using $\chi^{2}$ analysis and calculating odds ratios (OR). In vitro drug sensitivity was analyzed directly using $\mathrm{IC}_{50}$ as a continuous variable, as well as by dichotomizing isolates into "sensitive" and "resistant" categories based on $\mathrm{IC}_{50}$ values. For mefloquine, a resistant isolate had an $\mathrm{IC}_{50}$ above $20 \mathrm{ng} / \mathrm{mL}$ or an $\mathrm{IC}_{90}$ above $80 \mathrm{ng} / \mathrm{mL}$ and was categorized as sensitive if the $\mathrm{IC}_{50}$ and $\mathrm{IC}_{90}$ were below these values.

The relationship between molecular markers and in vitro sensitivity and the association between clinical outcomes and in vitro susceptibility was performed using $\chi^{2}$ analysis and $t$ tests. The relationship between molecular markers and clinical outcome was assessed by calculating relative risks (RR) and performing Cox proportional hazards modeling. Associations with gene amplification and recrudescent outcomes were assessed using $\chi^{2}$ analyses. A two-sided Fisher's exact test was used when expected cell-sizes were 5 or less. Nonnormally distributed $\mathrm{IC}_{50}$ values were normalized by natural $\log$ transformation.

\section{RESULTS}

Overall, 52 patients were enrolled into the study under mefloquine monotherapy. Demographic characteristics of the study participants are provided in Table 2. Twenty patients $(38.5 \%)$ failed mefloquine treatment over the course of the follow-up period and were given second-line therapy. The mean time to recrudescence was 35.7 days (Figure 1). Four recrudescences occurred in less than 14 days, six occurred between 14 and 28 days, and 10 occurred between days 29 and 42 of follow-up. There were no differences between recrudescent and nonrecrudescent patients in age, gender, parasitemia, or self-reported symptoms (Table 2). Parasitemia was 
TABLE 2

Demographic characteristics, enrollment genotype, and selected symptoms from medical history for 52 patients receiving mefloquine monotherapy

\begin{tabular}{|c|c|c|c|c|}
\hline & Overall & Recrudescent & Nonrecrudescent & $P$ value* \\
\hline Total, $N(\%)$ & 52 & $20(38.5)$ & $32(61.5)$ & - \\
\hline Female, $N(\%)$ & $12(23.1)$ & 7 (21.9) & $5(25.0)$ & 1.00 \\
\hline Age $($ mean $\pm \mathrm{SD})$ & $28.9 \pm 10.4$ & $30.0 \pm 11.8$ & $28.2 \pm 9.6$ & $0.54 \dagger$ \\
\hline Parasitemiał (median; SD) & 29,$040 ; 98,294$ & 45,$760 ; 119,002$ & 22,$440 ; 83,898$ & $0.23 \dagger$ \\
\hline Parasitemia range (min-max) & $16-550,000$ & $80-550,000$ & $16-356,400$ & \\
\hline Genotype I/III, $N(\%)$ & $44(84.6)$ & $18(94.7)$ & $27(90.0)$ & \\
\hline Genotype II/IV, $N(\%)$ & $6(11.5)$ & $1(5.3)$ & $3(10.0)$ & $1.00^{*}$ \\
\hline Chills, $N(\%)$ & $49(94.2)$ & $18(90.0)$ & $31(96.9)$ & 0.55 \\
\hline Cough, $N(\%)$ & $18(34.6)$ & $7(35.0)$ & $11(34.4)$ & 1.00 \\
\hline Diarrhea, $N(\%)$ & $2(3.9)$ & $1(5.0)$ & $1(3.1)$ & 1.00 \\
\hline Malaise, $N(\%)$ & $40(76.9)$ & $16(80.0)$ & $24(46.2)$ & 0.75 \\
\hline Vomiting, $N(\%)$ & $23(44.2)$ & $9(45.0)$ & $14(43.8)$ & 1.00 \\
\hline
\end{tabular}

analyzed as a predictor of recrudescence by several methods, including rank-sum and using different cutoffs (the 75 th percentile and 40,000 parasites/ $\mu \mathrm{L})$, and no significant relationship was found.

Blood samples from 2 of 52 patients did not yield quality DNA, but adequate DNA was extracted from the culture of one of these samples. Additionally, two blood samples yielded genotype patterns other than the expected categories and were excluded from categorical analyses, for a final analytic sample size of 49 . Of these, 18 were Category I genotypes $(36.7 \%), 27$ were Category III genotypes (55.1\%), 3 were Category II genotypes $(6.1 \%)$, and 1 was a Category IV genotype $(2.0 \%)$. In total, at the initial visit there were 4 isolates with Tyr86 (7.8\%), 24 with Phe184 (47.1\%), and 6 isolates with a mixed genotype at position $184(11.8 \%)$. There were no Cys1034, only one with Asn1042 (2.0\%), and one mixed genotype at position $1042(2.0 \%)$.

One of the genotypes falling outside the expected categories was a mixed tyrosine/phenylalanine phenotype at position 184 with a mixed aspartate/asparagine phenotype at position 1042. The other sample falling outside expected combinations had Tyr86 and a mixed tyrosine/phenylalanine phenotype at position 184. Of the three excluded samples (two with unexpected genotype patterns, one with poor quality DNA), one was a patient who eventually recrudesced and two were patients with successful malaria treatment.

pfmdrl and in vitro drug resistance. $\mathrm{IC}_{50}$ data were available for 42 patients: 26 were patients with successful mefloquine therapy and 16 were patients who recrudesced. Effective sample size was 40 , because two observations with

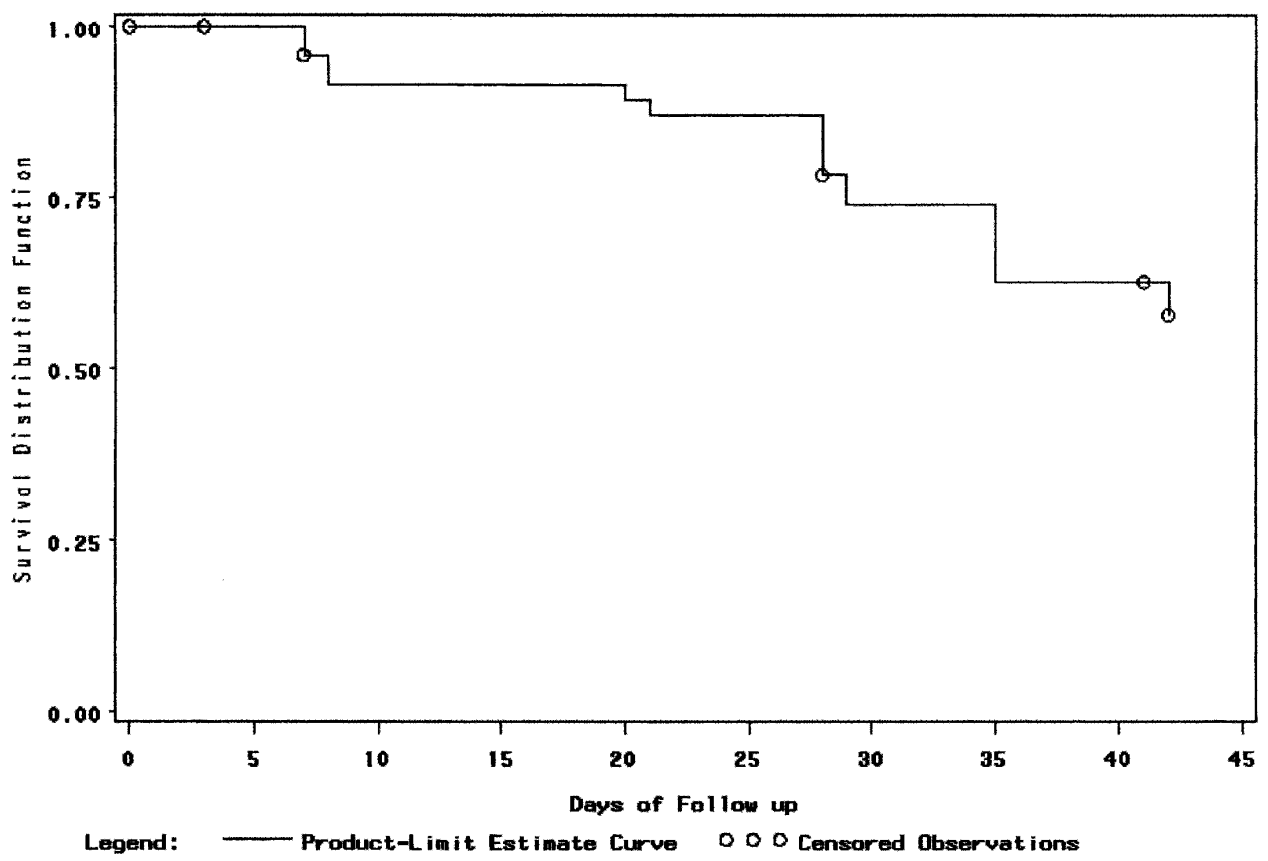

FIGURE 1. Kaplan-Meier survival curve for time to recrudescence in a population of falciparum malaria patients receiving mefloquine monotherapy, Sangkhlaburi, Thailand, 2001-2002. Survival rates are as follows: Day 7, 44 of 46; Day 8, 38 of 42; Day 20, 36 of 41; Day 21, 34 of 40; Day 26, 25 of 35; Day 29, 21 of 33; Day 35, 11 of 28; Day 42, 5 of 24. 
available $\mathrm{IC}_{50}$ were among the excluded patients (one successfully treated and one recrudescent). None of the individual polymorphisms observed (Tyr86, Phe184, and Asp1042) had a significant association with higher $\mathrm{IC}_{50}$ than the wild-type allele (Asn86, Tyr86, and Asn1042) when compared by $t$ test. $\mathrm{IC}_{50} \mathrm{~s}$ were also compared across genotype categories with the wild type (Category I) as the referent pattern; no significant associations were observed, although sample sizes in Categories II and IV were too small to be meaningful.

The mean mefloquine $\mathrm{IC}_{50}$ among isolates with the $p f m d r 1$ Category I/III $(N=36)$ genotype was $45.9 \mathrm{ng} / \mathrm{mL}$ ( $95 \%$ confidence interval [CI] 38.0-53.8) and $27.1 \mathrm{ng} / \mathrm{mL}$ (95\% CI $14.3-$ 39.9) among isolates with the Category II/IV genotype $(N=$ 4; $P=0.1226)$. Log-transformed $\mathrm{IC}_{50}$ s for chloroquine and quinine were not different between genotype Categories I/III versus Categories II/IV. Thus, $p f m d r 1$ genotype patterns determined directly from blood samples did not predict in vitro drug sensitivity using a continuous scale.

$\mathrm{IC}_{50}$ was also evaluated as a dichotomous outcome, using standard $\mathrm{IC}_{50}$ cutoffs to categorize each $\mathrm{IC}_{50}$ as "resistant" or "sensitive." According to the resistance $\mathrm{IC}_{50}$ cutoffs, 37 $(88.1 \%)$ of the 42 isolates were resistant to mefloquine, 21 $(50.0 \%)$ were resistant to chloroquine, and none were resistant to quinine. No individual polymorphism or category of genotype pattern had a significant association with dichotomous in vitro outcome compared with the others. Fisher's exact test was performed to determine if equal proportions of drug-resistant isolates fell into the Categories I/III versus Categories II/IV. There were no differences in level of mefloquine- or chloroquine-resistant isolates between bloodderived genotype Categories I/III and II/IV.

In contrast, $p f m d r 1$ genotype determined from the cultured parasite did predict in vitro $\mathrm{IC}_{50}$. Previous work done by our group found a $17 \%$ discordance between the genotype determined from blood and the genotype determined from cultured parasites. ${ }^{19}$ In the current data, when culture-derived genotypes were analyzed, there was a significant difference in the mean mefloquine sensitivity between categories of pfmdr1 polymorphisms. Although mean $\mathrm{IC}_{50} \mathrm{~s}$ did not differ substantially from blood data, the number of isolates in each category was different using data from culture. Category I/III isolates $(N=33)$ had a mean mefloquine $\mathrm{IC}_{50} 46.9 \mathrm{ng} / \mathrm{mL}$ (versus 45.9 in blood DNA analysis), and Category II/IV isolates $(N=6)$ had a significant difference in the mean mefloquine $\mathrm{IC}_{50} 27.7 \mathrm{ng} / \mathrm{mL}$ (versus 27.19 in blood DNA analysis, $P=0.0005)$. This suggests that culturing parasites may yield genotype changes that might be linked to in vitro resistance.

Association between in vitro and in vivo drug resistance. Student $t$ tests were conducted on continuous $\mathrm{IC}_{50}$ data to evaluate the difference in the laboratory $\mathrm{IC}_{50}$ between those who recrudesced and those who did not recrudesce. There were no significant differences in $\mathrm{IC}_{50}$ by recrudescence status for any of the drugs tested (mefloquine $P=0.4048$, chloroquine $P=0.2039$, quinine $P=0.3060$ ).

$\mathrm{IC}_{50}$ was also considered as a dichotomous outcome, as in the genetic analysis. Fisher's exact test was performed to determine if the proportions of in vitro drug-resistant isolates that had recrudescent and nonrecrudescent outcomes were equal. There were no differences in the $\mathrm{IC}_{50}$ level of mefloquine- or chloroquine-resistant isolates by recrudescence status. The sensitivity of the in vitro test to detect resistance, with the in vivo result as a gold standard, was $93.8 \%$; however, the specificity was only $15.4 \%$. The kappa statistic for agreement between these two measures was 0.07 , which was not significantly different from zero (no agreement, two-sided $P=$ $0.6332)$.

pfmdr1 polymorphisms as a predictor of clinical drug resistance. Polymorphisms in $p f m d r 1$ as determined using DNA extracted from blood were analyzed as predictors of clinical resistance (recrudescence). Differences in clinical resistance were analyzed by the polymorphism categories. At the initial clinic visit, 45 members of the mefloquine-monotherapy cohort $(91.8 \%)$ ) were infected with Category I/III parasites. Four $(8.2 \%)$ were infected with Category II/IV parasites. Eighteen of 45 Category I/III patients $(40.0 \%)$ and 1 of 4 Category II/IV patients $(25.0 \%)$ had recrudesced by the end of followup. The relative risk of recrudescence or treatment failure with Category I/III genotypes compared with Category II/IV genotypes was 1.60 (95\% CI $0.28,9.07)$. Thus, $p f m d r 1$ polymorphisms do not predict recrudescence after mefloquine monotherapy. The relative risks for in vivo resistance with genotype exposure are shown in Table 3.

Comparison between $\boldsymbol{p} \boldsymbol{f m} \boldsymbol{d r} \mathbf{1}$ genotypes on enrollment and recrudescence. Genotype data were available on 14 recrudescent patients at the time of recrudescence. None of the 14 patients had any polymorphism at position 86 or position 1034, both at initial visit and recrudescence. For 11 patients, pfmdr1 genotypes were identical on enrollment and on recrudescence. For three patients with mixed genotype on enrollment, monoclonal genotypes were found on recrudescence.

Gene copy number. At the initial visit, gene amplification determined from blood-extracted DNA was analyzed as a predictor of recrudescence. Five patients had approximately a 2-fold elevation in pfmdrl copy, and four of these patients recrudesced. Of the 46 patients who did not have elevated pfmdr1 gene copy number, 16 recrudesced, for a relative risk of 2.30 (95\% CI 1.27-4.15; Table 3). These results indicate that infection with at least a 2-fold increase in $p f m d r 1$ copy number may increase the risk of recrudescence.

\section{TABLE 3}

Relative risk of recrudescence for individual SNPs, categories of polymorphism patterns, and level of gene amplification for patients receiving mefloquine monotherapy for malaria on the ThaiMyanmar border (blood-derived data are shown)

\begin{tabular}{lrcc}
\hline & $N$ & RR & $(95 \% \mathrm{CI})$ \\
\hline Gene amplification & & & \\
$\quad$ None & 46 & Ref & - \\
2-fold & 5 & 2.30 & $(1.27-4.15)$ \\
3-fold & 0 & - & - \\
SNPs & & & \\
$\quad$ Tyr86 & 3 & 0.62 & $(0.11,3.50)$ \\
Phe184 & 24 & 0.88 & $(0.40,1.92)$ \\
Ser1034 & 0 & - & - \\
Asp1042 & 1 & $*$ & - \\
Categories & & & \\
I & 18 & 1.01 & $(0.31,3.32)$ \\
II & 3 & 0.85 & $(0.17,4.39)$ \\
III & 27 & 1.12 & $(0.55,2.29)$ \\
IV & 1 & $*$ & - \\
II/IV & 3 & Ref & - \\
I/III & 45 & 1.60 & $(0.28,9.07)$ \\
\hline
\end{tabular}

SNPs, single nucleotide polymorphisms; CI, confidence interval; RR, relative risk; Ref, referent level.

* Not calculable; no patients who failed therapy were infected with parasites that exhibited the trait of interest. 
pfmdr1 copy number was also determined for 10 patients upon recrudescence (Figure 2). Two patients had increased gene copy number at recrudescence. One patient had parasites with 2-fold increase in copy number at both admission and recrudescence. Parasites from the other patient did not have increased pfmdrl copy number on admission but had 2-fold increased copy number on recrudescence.

\section{DISCUSSION}

As rates of drug-resistant malaria rise, research into the mechanisms contributing to the development of drug resistance and ways to prevent this from occurring becomes more and more vital. Thailand is a country with a successful malaria control program but which suffers from multidrug-resistant malaria strains at its borders due to fluctuating populations with little malaria immunity. Because of the use of mefloquine monotherapy as first-line therapy in parts of the country, at the time of the study Thailand was in an ideal region to research the role of the $P$. falciparum gene pfmdrlin mefloquine drug resistance.

In this study, we investigated whether $p f m d r 1$ polymorphisms were predictive of drug resistance in vivo. In a cohort of patients treated with mefloquine monotherapy, we found that $p f m d r 1$ genotype determined on patient blood was not statistically associated with mefloquine, chloroquine, or quinine $\mathrm{IC}_{50} \mathrm{~s}$. However, when genotype was determined on DNA from cultured parasites, a significant association between $p f m d r 1$ genotype categories and mefloquine $\mathrm{IC}_{50}$ was observed. These results indicate that selection of $p f m d r 1$
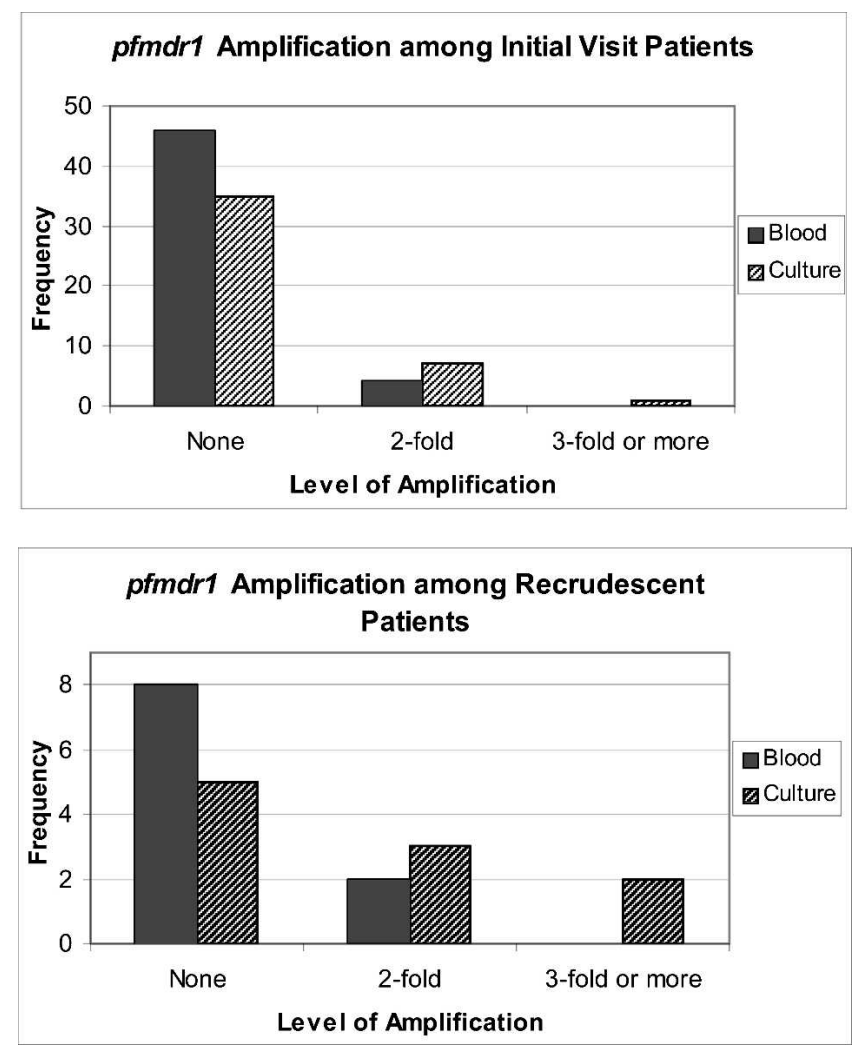

FIGURE 2. Frequency of $p f m d r 1$ gene amplification among patients receiving mefloquine monotherapy for malaria at the ThaiMyanmar border. genotypes may occur when parasites become adapted to culture. This might explain another observation made in this paper, namely that there was a poor correlation between in vitro and in vivo drug resistance. Polymorphisms among parasites that have been adapted to culture might be expected to correlate more closely with resistance determined from in vitro test than resistance determined from clinical outcome.

Clinically, we found a slightly increased risk of recrudescence with the resistant $p f m d r 1$ genotype Categories I and III (RR 1.60), which was statistically non-significant. However, increased $p f m d r 1$ copy number was predictive of recrudescence. Those with a 2 -fold increase in copy number at the initial visit were more likely to recrudesce, with a relative risk of recrudescence of 2.30 compared with those with no $\mathrm{pfmdr} 1$ amplification (95\% CI 1.27-4.15). This result affirms laboratory studies of parasite isolates, which have found increased expression of pfmdr1 mRNA in mefloquine-resistant strains compared with mefloquine-sensitive strains. ${ }^{20-22}$ In a rodent model of malaria, the putative $p f m d r 1$ homologue was 2- to 3 -fold amplified in rodents infected with mefloquine-resistant malaria strains. ${ }^{23}$ Studies in Thailand and other Southeast Asian countries have shown an association between increased gene copy number and in vitro resistance to mefloquine, ${ }^{10,24}$ with conflicting evidence about the independent role of polymorphismss at codon 86. Other studies in Thailand and elsewhere, however, did not observe a relationship between drug resistance and gene amplification. ${ }^{14,25}$ This could indicate differing mechanisms of resistance in different geographical areas or that results of studies examining gene copy number may be different depending on the background antimalarial use in the region. Evaluation of the expression of $p f m d r 1$ during human infection may be an important next step in resolving its role in mefloquine drug resistance.

The methods developed by our group ${ }^{19}$ were designed to detect amplification at specific levels of confidence. The $90 \%$ confidence level corresponded to a $p f m d r 1: l d h$ copy number ratio of 1.7 to 1 ; thus, at $90 \%$ confidence we could detect approximately a 2 -fold level of amplification. At the $95 \%$ confidence level, the pfmdr1:ldh ratio was 3.05 to 1 , thus we could detect 3 -fold amplification with $95 \%$ confidence. However, no isolates exhibited 3 -fold amplification at the initial clinic visit, so the more sensitive 2-fold detection was used. These methods can be performed on parasite DNA extracted directly from blood samples, and the technology is not only effective but is not unaffordable for low-income countries.

In our data, gene copy numbers determined for cultured parasites did not have good agreement with gene copy numbers determined directly from patient blood, agreeing on only $25 \%$ of samples (data not shown). Most studies of molecular markers for drug resistance have focused on traditional in vitro resistance measures involving culture or have used DNA from cultured parasites. The correlation between in vitro and in vivo outcomes has not been established in these settings. The genotype determined from a cultured isolate may not be the same genotype as the predominant infecting strain, as adaptation to culture may select for one strain of a multiclonal infection or may induce changes in the parasite genotype. In our study, we found that the in vitro test had $93 \%$ sensitivity and $15 \%$ specificity for detecting mefloquine treatment failure. By contrast, a comparison of in vitro and in vivo chloroquine resistance in Cameroon found the in vitro test to have $67 \%$ sensitivity and $84 \%$ specificity, with a kappa 
statistic for agreement between the two measures that was much higher than the one presented here ( 0.48 versus 0.07$){ }^{26}$ Additionally, the positive predictive value for in vitro resistance in our study was only $40.5 \%$. Other strong methodologic literature in this area is lacking. The disparity between in vivo and in vitro resistance could be due to evolutionary selection in culture or other confounding factors such as differences in drug metabolism, or preexisting antimalarial immunity could also contribute. However, there remains a lack of research about the proper interpretation of in vitro resistance measures as applied to in vivo outcomes.

Previous work done by our group found that the main factor in predicting recrudescence in hospitalized patients under artesunate therapy was the initial parasite burden of the patient. ${ }^{27}$ However, the current study found no relationship between parasite burden and recrudescence. The conflicting results of our two studies could be due to several reasons. The use of hospitalized patients in the previous study reflects a more severe malaria infection, and patients might be expected to have more extreme parasite burdens. The current study has a small sample size, but the pharmacokinetics of the malaria therapy used are also different. Mefloquine, used in the current project, has a long half-life and is effective against blood parasite levels for a longer period of time. Artesunate, however, is quickly metabolized; patients with higher parasite burden may recrudesce more because the blood drug level does not remain high enough to affect the entire parasite population.

Our data are consistent with other studies of pfmdr1 molecular markers as predictors of mefloquine treatment failure. Two studies ${ }^{28,29}$ found that $p f m d r 1$ point mutations did not predict recrudescence. One of these studies ${ }^{29}$ also found that pfmdrl gene amplification was associated with mefloquine failure. Another study was unable to analyze polymorphisms as predictors of recrudescence because mefloquine treatment was efficacious in the study area. ${ }^{16}$

Due to the prospective study design, this study has many strengths. We were able to evaluate $p f m d r 1$ genotype both before treatment and at the time of recrudescence. Patients were closely followed for recrudescence with regular blood smears. The setting of this study also gave reassurance against the possibility for reinfection, as the immediate Sangkhlaburi District carries a very low risk for malaria, and patients must venture further into the jungle to increase their risk. This study also has several analytical advantages, including realtime PCR technology developed to rapidly detect polymorphisms, the ability to test both blood and culture parasites from patients for polymorphisms, and the availability of $\mathrm{IC}_{50}$ data. This study is the second study to our knowledge to evaluate $p f m d r 1$ polymorphisms and drug resistance in human populations, and is the first in Southeast Asia, which has proved to be a hot spot for the development of resistance.

However, there were also several drawbacks in conducting this study, the greatest of which is the small sample size. Mefloquine treatment failure records at malaria clinics and preliminary data from this study encouraged the Thai Ministry of Public Health to change the first-line malaria treatment regimen from mefloquine alone to a mefloquine-artesunate combination, thus cutting the study short and limiting power. Additionally, other factors contributing to drug resistance and/or recrudescence were not measured, such as immune status, individual nutritional factors, and drug metabolism. Despite the evidence in the literature that $p f m d r l$ is correlated with mefloquine drug resistance, there could be another gene linked to $p f m d r 1$ that is actually responsible for resistance. Although the risk of reinfection in this population is low, strain typing was not performed; therefore, reinfection cannot be ruled out. However, any misclassification that might have labeled a truly reinfected individual as a recrudescent individual would have been nondifferential across genotype exposure groups, resulting in a bias toward the null.

Our results suggest that $p f m d r 1$ gene polymorphisms are probably not a useful surveillance tool for detecting drugresistant malaria in Thailand; however, the relationship between gene amplification and recrudescence may be useful in evaluating potential for malaria recrudescence.

The use of real-time PCR allowing for direct detecting of potential polymorphisms could be an appropriate technology for study of molecular markers of antimalarial resistance, and polymorphisms in pfmdrl deserve further investigation. It may be difficult to repeat this study because there are few endemic areas in the world where mefloquine monotherapy is used today. However, application of the technology to evaluate resistance development to artemisinin-based combination therapy (ACT), which has mefloquine or its related compound, lumefantrine, as a partner drug, would be of interest, as $p f m d r 1$ has been shown in vitro to be associated with reduced artemisinin sensitivity as well. ${ }^{17}$ Because ACTs are being introduced in many endemic areas, especially in subSaharan Africa, real-time PCR-based surveillance for $p \mathrm{fmdr1}$ polymorphisms could become an important public health tool.

Received June 16, 2004. Accepted for publication October 28, 2004.

Acknowledgments: Many thanks go to the nursing and laboratory staff at AFRIMS for contributing their expertise and for their daily dedication to the populations they serve. Thanks also to Dr. Kanungnit Congpuong, the staff of KRCH, and the Vector Borne Diseases Control Unit No. 9 (Sangkhlaburi) for their assistance and support.

Financial support: This work was supported by NIH grant R23 AI054590 and U.S. Department of Defense-Global Emerging Infections Surveillance and Response Program (DoD-GEIS).

Authors' addresses: Amy L. Nelson, University of North Carolina Department of Epidemiology, McGavran-Greenberg Hall, Chapel Hill, NC 27599-7435, 2358 Antiqua Ct., Reston, VA 20191, Telephone: 919-966-7414. Anne Purfield, University of North Carolina School of Medicine, Department of Microbiology and Immunology, Mary Ellen Jones Building, Chapel Hill, NC 27599, Telephone: 919843-4384, Fax: 919-966-2089. Philip McDaniel, 10803 SE Cherry Blossom Dr., Portland, OR 97216, Telephone: 503-261-7200, Fax: 503261-7226; Nichipat Uthaimongkol, AFRIMS, 315/6 Rajvithi Rd., Bangkok 10400, Thailand. Nillawan Buathong, AFRIMS, 315/6 Rajvithi Rd., Bangkok 10400, Thailand. Sabaitip Sriwichai, AFRIMS, 315/6 Rajvithi Rd, Bangkok 10400, Thailand. R. Scott Miller, AFRIMS, 315/6 Rajvithi Rd., Bangkok 10400, Thailand. Chansuda Wongsrichanalai, U.S. Naval Medical Research Unit No. 2 (NAMRU-2), Jakarta, Indonesia. Steven R. Meshnick, University of North Carolina School of Public Health, McGavran Greenberg Hall, Chapel Hill, NC 27599-7435, Telephone: 919-966-7414 or 843-4385, Fax: 919-966-2089.

\section{REFERENCES}

1. RBM, What is Malaria? World Health Organization Roll Back Malaria. WHO, Geneva, Switzerland.

2. D'Alessandro U, 1998. Antimalarial drug resistance: surveillance and molecular methods for national malaria control programmes. Mem Inst Oswaldo Cruz 93: 627-630. 
3. Wellems TE, Plowe CV, 2001. Chloroquine-resistant malaria. $J$ Infect Dis 184: 770-776.

4. Fidock DA, Nomura T, Talley AK, Cooper RA, Dzekunov SM, Ferdig MT, Ursos LM, Sidhu AB, Naude B, Deitsch KW, Su XZ, Wootton JC, Roepe PD, Wellems TE, 2000. Mutations in the P. falciparum digestive vacuole transmembrane protein PfCRT and evidence for their role in chloroquine resistance. Mol Cell 6: 861-871.

5. Adagut IS, Warhurst DC, 2001. Plasmodium falciparum: linkage disequilibrium between loci in chromosomes 7 and 5 and chloroquine selective pressure in Northern Nigeria. Parasitology 123: 219-224.

6. Chen N, Russell B, Staley J, Kotecka B, Nasveld P, Cheng Q, 2001. Sequence polymorphisms in pfcrt are strongly associated with chloroquine resistance in Plasmodium falciparum. J Infect Dis 183: 1543-1545.

7. Djimde A, Doumbo OK, Cortese JF, Kayentao K, Doumbo S, Diourte Y, Dicko A, Su XZ, Nomura T, Fidock DA, Wellems TE, Plowe CV, Coulibaly D, 2001. A molecular marker for chloroquine-resistant falciparum malaria. $N$ Engl J Med 344: 257-263.

8. Babiker HA, Pringle SJ, Abdel-Muhsin A, Mackinnon M, Hunt P, Walliker D, 2001. High-level chloroquine resistance in Sudanese isolates of Plasmodium falciparum is associated with mutations in the chloroquine resistance transporter gene pfcrt and the multidrug resistance Gene pfmdr1. J Infect Dis 183: $1535-1538$.

9. Adagu IS, Warhurst DC, 1999. Association of cg2 and pfmdr1 genotype with chloroquine resistance in field samples of Plasmodium falciparum from Nigeria. Parasitology 119: 343-348.

10. Price RN, Cassar C, Brockman A, Duraisingh M, van Vugt M, White NJ, Nosten F, Krishna S, 1999. The pfmdr1 gene is associated with a multidrug-resistant phenotype in Plasmodium falciparum from the western border of Thailand. Antimicrob Agents Chemother 43: 2943-2949.

11. Zalis MG, Pang L, Silveira MS, Milhous WK, Wirth DF, 1998. Characterization of Plasmodium falciparum isolated from the Amazon region of Brazil: evidence for quinine resistance. Am J Trop Med Hyg 58: 630-637.

12. Dorsey G, Kamya MR, Singh A, Rosenthal PJ, 2001. Polymorphisms in the Plasmodium falciparum pfcrt and pfmdr-1 genes and clinical response to chloroquine in Kampala, Uganda. $J$ Infect Dis 183: 1417-1420.

13. Pillai DR, Labbe AC, Vanisaveth V, Hongvangthong B, Pomphida S, Inkathone S, Zhong K, Kain KC, 2001. Plasmodium falciparum malaria in Laos: chloroquine treatment outcome and predictive value of molecular markers. J Infect Dis 183: 789-795.

14. Chaiyaroj SC, Buranakiti A, Angkasekwinai P, Looressuwan S, Cowman AF, 1999. Analysis of mefloquine resistance and amplification of pfmdr1 in multidrug-resistant Plasmodium falciparum isolates from Thailand. Am J Trop Med Hyg 61: 780783.

15. Povoa MM, Adagu IS, Oliveira SG, Machado RL, Miles MA, Warhurst DC, 1998. Pfmdr1 Asn1042Asp and Asp1246Tyr polymorphisms, thought to be associated with chloroquine resistance, are present in chloroquine-resistant and -sensitive Brazilian field isolates of Plasmodium falciparum. Exp Parasitol 88: 64-68.

16. Pillai DR, Hijar G, Montoya Y, Marouino W, Ruebush TK 2nd,
Wongsrichanalai C, Kain KC, 2003. Lack of prediction of mefloquine and mefloquine-artesunate treatment outcome by mutations in the Plasmodium falciparum multidrug resistance 1 (pfmdr1) gene for P. falciparum malaria in Peru. Am J Trop Med Hyg 68: 107-110.

17. Pickard AL, Wongsrichanalai C, Purfield A, Kamwendo D, Emery K, Zalewski C, Kawamoto F, Miller RS, Meshnick SR, 2003. Resistance to antimalarials in Southeast Asia and genetic polymorphisms in pfmdr1. Antimicrob Agents Chemother 47: 2418-2423.

18. Webster HK, Boudreau EF, Pavanand K, Yongvanitchit K, Pang LW, 1985. Antimalarial drug susceptibility testing of Plasmodium falciparum in Thailand using a microdilution radioisotope method. Am J Trop Med Hyg 34: 228-235.

19. Purfield A, Nelson A, Laoboonchai A, Congpuong K, McDaniel P, Miller RS, Welch K, Wongsrichanalai C, Meshnick SR, 2004. A new method for detection of $p f m d r 1$ mutations in Plasmodium falciparum DNA using real time PCR. Malaria 3(1): 9-15.

20. Cowman AF, Galatis D, Thompson JK, 1994. Selection for mefloquine resistance in Plasmodium falciparum is linked to amplification of the pfmdr1 gene and cross-resistance to halofantrine and quinine. Proc Natl Acad Sci U S A 91: 1143-1147.

21. Kim HS, Okuda Y, Begum K, Nagai Y, Wataya Y, Kimura M, Huruta T, 2001. Analysis of Pfmdr 1 gene in mefloquineresistant Plasmodium falciparum. Nucleic Acids Res (Suppl): 231-232.

22. Begum K, Kim HS, Okuda Y, Wataya Y, Kimura M, Huruta T, 2002. Genomic analysis of mefloquine-resistant Plasmodium falciparum. Nucleic Acids Res 2 (Suppl 30): 223-224.

23. Gervais GW, Trujillo K, Robinson BL, Peters W, Serrano AE, 1999. Plasmodium berghei: identification of an mdr-like gene associated with drug resistance. Exp Parasitol 91: 86-92.

24. Wilson CM, Volkman SK, Thaithong S, Martin RK, Kyle DE, Milhous WK, Wirth DF, 1993. Amplification of pfmdr 1 associated with mefloquine and halofantrine resistance in Plasmodium falciparum from Thailand. Mol Biochem Parasitol 57: 151-160.

25. Basco LK, Le Bras J, Rhoades Z, Wilson CM, 1995. Analysis of pfmdr1 and drug susceptibility in fresh isolates of Plasmodium falciparum from subsaharan Africa. Mol Biochem Parasitol 74: 157-166.

26. Ringwald P, Basco LK, 1999. Comparison of in vivo and in vitro tests of resistance in patients treated with chloroquine in Yaounde, Cameroon. Bull World Health Org 77: 34-43.

27. Ittarat $\mathrm{W}$, Pickard $\mathrm{AL}$, Rattanasinganchan $\mathrm{P}$, Wilairatana $\mathrm{P}$, Looareesuwan S, Emery K, Low J, Udomsangpetch R, Meshnick SR, 2003. Recrudescence in artesunate-treated patients with falciparum malaria is dependent on parasite burden not on parasite factors. Am J Trop Med Hyg 68: 147-152.

28. Mawili-Mboumba DP, Kun JF, Lell B, Kremsner PG, Ntoumi F, 2002. Pfmdr1 alleles and response to ultralow-dose mefloquine treatment in Gabonese patients. Antimicrob Agents Chemother 46: 166-170.

29. Price RN, Uhlemann AC, Brockman A, McGready R, Ashley E, Phaipun L, Patel R, Laing K, Looareesuwan S, White NJ, Nosten F, Krishna S, 2004. Mefloquine resistance in Plasmodium falciparum and increased pfmdr1 gene copy number. Lancet 364: 438-447. 